Proc. Indian Acad. Sci. (Math. Sci.), Vol. 101, No. 3, December 1991, pp. 169-177.

(C) Printed in India.

\title{
A multiplier theorem for the sublaplacian on the Heisenberg group
}

\author{
S THANGAVELU \\ TIFR Centre, Indian Institute of Science Campus, Bangalore 560012, India \\ MS received 24 January 1990
} Abstract. A multiplier theorem for the sublaplacian on the Heisenberg group is proved using
Littlewood-Paley-Stein theory of $g$-functions.

Keywords. Multiplier theorem; sublaplacian; Heisenberg group; Littlewood-Paley-Stein theory; $g$-functions.

\section{Introduction}

Consider the Heisenberg group $H_{n}$ and the sublaplacian $\mathscr{L}$ on $H_{n} . \mathscr{L}$ is a formally non-negative hypoelliptic differential operator which has a unique self-adjoint extension to $L^{2}\left(H_{n}\right)$. If $\varphi$ is a function defined on $\mathbb{R}$ then using spectral theorem one can define the operator $\varphi(\mathscr{L})$. If $\varphi$ is a bounded function, then $\varphi(\mathscr{L})$ will be bounded on $L^{2}\left(H_{n}\right)$. In the same spirit one likes to find sufficient conditions on $\varphi$ so that the operator $\varphi(\mathscr{L})$ will be bounded on $L^{p}\left(H_{n}\right)$.

This problem was studied by Mauceri [4] and the following result was proved.

If the function $\varphi$ is $n+3$ times differentiable and satisfies the estimate $\left|\varphi^{(k)}(t)\right| \leqslant C(1+|t|)^{-k}, k=0,1, \ldots(n+3)$, then $\varphi(\mathscr{L})$ is bounded operator on $L^{p}\left(H_{n}\right)$ for all $1<p<\infty$.

This result was proved using the theory of singular integrals on homogeneous spaces developed by Coifman and Weiss [1]. Later Mauceri improved the above result replacing the smoothness condition on $\varphi$ by a fractional order condition of the order $s>n+2$ (see [5]). Here we propose to give a different proof of the multiplier theorem. We prove:

Theorem. Let $\varphi$ be $v$ times differentiable and satisfies $\left|\varphi^{(k)}(t)\right| \leqslant C(1+|t|)^{-k}$ for $k=0,1, \ldots v$ where $v=n+2$ if $n$ is even and $v=n+3$ if $n$ is odd. Then $\varphi(\mathscr{L})$ is a bounded operator on $L^{p}\left(H_{n}\right), 1<p<\infty$.

Our proof of this theorem is based on Littlewood-Paley-Stein theory of $g_{k}$ and $g_{k}^{*}$ functions. We adapt this method which was originally employed by Stein [6] to prove the Hormander-Mihlin multiplier theorem for the Fourier transform, to the present case. The same technique was successfully employed by Strichartz [7] and by the author [9], [10] to prove some multiplier theorems. One good thing about this approach is that the proof is simple and also we get a sharper result when $n$ is even. 


\section{Preliminaries}

The main reference for this section is [3]. See also [4]. The $(2 n+1)$-dimensional Heisenberg group $H_{n}$ is the nil potent Lie group whose underlying manifold is $\mathbb{C}^{n} \times \mathbb{R}$. The group structure is given by

$$
(z, t)(\xi, s)=(z+\xi, t+s+2 \operatorname{Im} z \cdot \bar{\xi})
$$

where $t, s \in \mathbb{R}$ and $z, \xi \in \mathbb{C}^{n}$. The Haar measure on $H_{n}$ is simply the Lebesgue measure $\mathrm{d} z \mathrm{~d} s$ on $\mathbb{C}^{n} \times \mathbb{R}$. For $w=(z, s)$ the homogeneous norm $|w|$ is defined by $|w|^{4}=|z|^{4}+s^{2}$.

We next recall the definition of the Fourier transform on $H_{n}$. The infinite dimensional representations of $H_{n}$ are parametrized by $\mathbb{R} \backslash\{0\}$. If $\lambda \neq 0$, then all the representations $\pi_{\lambda}$ can be realized on the same Hilbert space $L^{2}\left(\mathbb{R}^{n}\right)$. For $(z, s) \in H_{n}, \pi_{\lambda}(z, s)$ is the operator acting on $L^{2}\left(\mathbb{R}^{n}\right)$ by the prescription

$$
\pi_{\lambda}(z, s) \varphi(\xi)=\exp (i \lambda s) \exp [i 2 \lambda(2 \xi-x) \cdot y] \varphi(\xi-x),
$$

where $z=x+i y$ and $\xi \in \mathbb{R}^{n}$.

The Fourier transform $\hat{f}$ of an $L^{1}$ function $f$ on $H_{n}$ is then the operator valued function

$$
\widehat{f}(\lambda)=\int_{H_{n}} f(w) \pi_{\lambda}(w) \mathrm{d} w .
$$

Then we have the following Plancherel formula:

$$
\|f\|_{2}^{2}=\frac{2^{n-1}}{\pi^{n+1}} \int|\lambda|^{n}\|\hat{f}(\lambda)\|_{H S}^{2} \mathrm{~d} \lambda,
$$

where \|\|$_{H S}$ is the Hilbert-Schmidt norm. We also have an inversion formula

$$
f(w)=\int \operatorname{tr}\left(\pi_{\lambda}(w)^{*} \hat{f}(\lambda)\right)|\lambda|^{n} \mathrm{~d} \lambda,
$$

where $\mathrm{tr}$ is the canonical semifinite trace.

For each $\lambda \neq 0$ we can select an orthonormal basis for $L^{2}\left(\mathbb{R}^{n}\right)$. Let $\Phi_{\alpha}^{\lambda}(x)=\left(2|\lambda|^{2}\right)^{n / 2} \Phi_{\alpha}\left(\left(2|\lambda|^{\frac{1}{2}} x\right)\right)$ where $\Phi_{\alpha}$ are the Hermite functions on $\mathbb{R}^{n}$. Then $\left\{\Phi_{\alpha}^{\lambda}\right\}$ is an orthonormal basis for $L^{2}\left\{\mathbb{R}^{n}\right)$. Let $P_{N}(\lambda)$ denote the projection of $L^{2}\left(\mathbb{R}^{n}\right)$ onto the eigenspace spanned by $\left\{\Phi_{\alpha}^{\lambda}:|\alpha|=N\right\}$. Using these operators $P_{N}(\lambda)$ we can write the Fourier transform of a zonal function in a simple way.

Let $f(z, s)=f(|z|, s)$ be a zonal function and $f(z, \lambda)$ be the Fourier transform in the $s$-variable.

$$
\tilde{f(z, \lambda)}=\int \exp (i \lambda s) f(z, s) \mathrm{d} s .
$$

Define $R_{N}(\lambda, f)$ by the formula

$$
R_{N}(\lambda, f)=C_{N} \frac{N !}{(N+n-1) !} \int_{0}^{\infty} \tilde{f}(r, \lambda) L_{N}^{n-1}\left(2|\lambda| r^{2}\right) \exp \left(-|\lambda| r^{2}\right) r^{2 n-1} \mathrm{~d} r,(7)
$$


where $L_{N}^{n-1}$ are the Laguerre polynomials of type $(n-1)$. Then one has

$$
\hat{f}(\lambda)=\sum_{N=0}^{\infty} R_{N}(\lambda, f) P_{N}(\lambda)
$$

And the Plancherel formula takes the form

$$
\|f\|_{2}^{2}=\frac{2^{n-1}}{\pi^{n+1}} \int \sum_{N=0}^{\infty}\left|R_{N}(\lambda, f)\right|^{2} \frac{(N+n-1) !}{N !}|\lambda|^{n} \mathrm{~d} \lambda .
$$

On $H_{n}$ consider the following left invariant vector fields.

$$
Z_{j}=\frac{\partial}{\partial z_{j}}+i \bar{z}_{j} \frac{\partial}{\partial t}, \bar{Z}_{j}=\frac{\partial}{\partial \bar{z}_{j}}-i z_{j} \frac{\partial}{\partial t} .
$$

The sublaplacian $\mathscr{L}$ is then defined by

$$
\mathscr{L}=-\frac{1}{2} \sum_{j=1}^{n}\left(Z_{j} \bar{Z}_{j}+\bar{Z}_{j} Z_{j}\right)
$$

Each representation $\pi_{\lambda}$ determines a Lie algebra representation $\mathrm{d} \pi_{\lambda}$. It can be shown that $\mathrm{d} \pi_{\lambda}(\mathscr{L})$ is a closable operator. Its closure is denoted by $H(\lambda)$ and it has the following spectral decomposition:

$$
H(\lambda)=\sum_{N=0}^{\infty}(2 N+n)|\lambda| P_{N}(\lambda)
$$

For any reasonable function $\varphi$ on $\mathbb{R}$, using spectral theorem, one can define the operator $\varphi(\mathscr{L})$. It can be shown that $\varphi(\mathscr{L})$ is a convolution operator with kernel $k$ i.e. $\varphi(\mathscr{L}) f=k * f$. The Fourier transform of $k$ is given by

$$
\hat{k}(\lambda)=\sum_{N=0}^{\infty} \varphi((2 N+n)|\lambda|) P_{N}(\lambda) .
$$

All these things will be made use of in the following sections.

\section{Littlewood-Paley-Stein theory on $H_{n}$}

In [2] Folland has shown that the sublaplacian $\mathscr{L}$ generates a contraction semigroup $T^{t}$ which satisfies all the conditions required to develop a Littlewood-Paley-Stein theory (see [6]). As in Stein [6] we define, for each positive integer $k$, the following functions

$$
\begin{aligned}
& \left(g_{k}(f, w)\right)^{2}=\int_{0}^{\infty} t^{2 k-1}\left|\partial_{t}^{k} T^{t} f(w)\right|^{2} \mathrm{~d} t \\
& \left(g_{k}^{*}(f, w)\right)^{2}=\int_{H_{n}} \int_{0}^{\infty} t^{-n}\left(1+t^{-2}|v|^{4}\right)^{-k}\left|\partial_{\eta}^{k} T^{t} f\left(v^{-1} w\right)\right|^{2} \mathrm{~d} t \mathrm{~d} v .
\end{aligned}
$$

For these functions we will prove the following theorem. 
Theorem 3.1. (i) For $k \geqslant 1,\left\|g_{k}(f)\right\|_{2}=2^{-k}\|f\|_{2}$.

(ii) For $1<p<\infty, C_{1}\|f\|_{p} \leqslant\left\|g_{k}(f)\right\|_{p} \leqslant C_{2}\|f\|_{p}$.

(iii) If $k>(n+1) / 2$ and $p>2$, then $\left\|g_{k}^{*}(f)\right\|_{p} \leqslant C\|f\|_{p}$.

Proof. The inequality $\left\|g_{k}(f)\right\|_{p} \leqslant C_{2}\|f\|_{p}$ follows from the general theory. The reverse inequality can be easily deduced once we have (i). When $k>(n+1) / 2$, the function $\left(1+|v|^{4}\right)^{-k}$ is integrable and hence one can prove (iii) using (i). This is routine and well known. So, it remains to prove (i).

We prove (i) when $k=1$. The case $k>1$ is similar. From the definition it follows that

$$
\left\|g_{1}(f)\right\|_{2}^{2}=\int_{0}^{\infty} \int_{H_{n}} t\left|\partial_{t} T^{t} f(w)\right|^{2} \mathrm{~d} w \mathrm{~d} t .
$$

In view of the Plancherel formula (4) the integral becomes

$$
\int_{H_{n}}\left|\partial_{t} T^{t} f(w)\right|^{2} \mathrm{~d} w=\frac{2^{n-1}}{\pi^{n-1}} \int|\lambda|^{n} \|\left(\partial_{t} T^{\mathrm{t} f} \hat{)}(\lambda) \|_{H S}^{2} \mathrm{~d} \lambda .\right.
$$

Since $T^{t} f=\exp (-t \mathscr{L}) f$, we see that

$$
\left(\partial_{t} T^{\dagger} f\right) \hat{(\lambda)}=-H(\lambda) \exp (-t H(\lambda)) \hat{f}(\lambda)
$$

and hence its squared Hilbert-Schmidt norm is given by the expression

$$
\sum_{\alpha}((2|\alpha|+n)|\lambda|)^{2} \exp (-2 t(2|\alpha|+n)|\lambda|)\left(\Phi_{\alpha}^{\lambda}, \hat{f}(\lambda)^{*} \hat{f}(\lambda) \Phi_{\alpha}^{\lambda}\right) .
$$

If we use this in (18) and integrate with respect to $t \mathrm{~d} t$, we will get

$$
\left\|g_{1}(f)\right\|_{2}^{2}=2^{-2} \frac{2^{n-1}}{\pi^{n+1}} \int|\lambda|^{n}\|\hat{f}(\lambda)\|_{H S}^{2} \mathrm{~d} \lambda
$$

And this proves that $\left\|g_{1}(f)\right\|_{2}=2^{-1}\|f\|_{2}$.

\section{The multiplier theorem}

Let us set $M f=\varphi(\mathscr{L}) f$. To prove the multiplier theorem what we need is the following pointwise inequality.

$$
g_{k+1}(M f) \leqslant C g_{k}^{*}(f)
$$

for some integer $k>(n+1) / 2$. For then the multiplier theorem for $p>2$ will follow immediately from Theorem 3.1. For $p<2$ one can use duality to conclude that $M$ is bounded on $L^{p}\left(H_{n}\right)$.

So, we proceed to prove the inequality (21). Let us set $u_{t}=T^{t} f, U_{t}=T^{t}(M f)$. Then it is easy to see that

$$
U_{t+s}(w)=\left(G_{t} * u_{s}\right)(w)
$$

where the Fourier transform of $G_{t}$ is given by

$$
\hat{G}_{t}(\lambda)=\sum_{N=0}^{\infty} \exp (-(2 N+n)|\lambda| t) \varphi((2 N+n)|\lambda|) P_{N}(\lambda)
$$


Differentiating (22) $k$ times with respect to $t$ and once with respect to $s$ and putting $t=s$ we obtain

$$
\partial_{t}^{k+1} T^{2 t}(M f)=F_{\mathrm{t}} * \partial_{t} T^{t} f
$$

where the Fourier transform of $F_{t}$ is given by

$$
\hat{F}_{t}(\lambda)=(-1)^{k} \sum_{N=0}^{\infty} \exp (-(2 N+n)|\lambda| t)(2 N+n)^{k}|\lambda|^{k} \varphi((2 N+n)|\lambda|) P_{N}(\lambda)
$$

Therefore, we have

$$
\left|\partial_{t}^{k+1} T^{2 t}(M f)(w)\right| \leqslant \int\left|F_{t}(v)\right|\left|\partial_{t} T^{t} f\left(v^{-1} w\right)\right| \mathrm{d} v .
$$

Applying Cauchy-Schwartz inequality

$$
\left|\partial_{t}^{k+1} T^{2 t}(M f)(w)\right|^{2} \leqslant A_{t} \cdot B_{t}(w),
$$

where we have written

$$
\begin{aligned}
A_{t} & =\int\left|F_{t}(v)\right|^{2}\left(1+t^{-2}|v|^{4}\right)^{k} \mathrm{~d} v \\
B_{t}(w) & =\int\left(1+t^{-2}|v|^{4}\right)^{-k}\left|\partial_{t} T^{t}\left(v^{-1} w\right)\right|^{2} \mathrm{~d} v .
\end{aligned}
$$

Now to complete the proof we need the estimate of the following Lemma.

Lemma. Under the hypothesis of the theorem the estimate $A_{t} \leqslant C t^{-n-2 k-1}$ is valid when $k$ is the smallest integer greater than $(n+1) / 2$.

Assuming the lemma for a moment it is easy to establish inequality (21). Indeed, from (26) we have

$$
\left|\partial_{t}^{k+1} T^{2 t}(M f)(w)\right|^{2} \leqslant C t^{-n-2 k-1} B_{t}(w) .
$$

Integrating this against $t^{2 k+1}$ we get

$$
g_{k+1}(M f, w) \leqslant C g_{k}^{*}(f, w) .
$$

This completes the proof of the multiplier theorem modulo the above lemma.

\section{Proof of the Lemma}

To prove the Lemma let us write

$$
\begin{aligned}
& I=\int_{|w| \leqslant \sqrt{t}}\left|F_{\mathrm{r}}(w)\right|^{2}\left(1+t^{-2}|w|^{4}\right)^{k} \mathrm{~d} w \\
& J=\int_{|w|>\sqrt{t}}\left|F_{t}(w)\right|^{2}\left(1+t^{-2}|w|^{4}\right)^{k} \mathrm{~d} w .
\end{aligned}
$$


Estimating the integral $I$ is easy. We note that since $|w| \leqslant \sqrt{t}$

$$
I \leqslant C \int\left|F_{t}(w)\right|^{2} \mathrm{~d} w
$$

and hence in view of Plancherel formula

$$
\begin{aligned}
I & \leqslant C \int|\lambda|^{n}\left(\sum_{N=0}^{\infty}(2 N+n)^{2 k}|\lambda|^{2 k} \exp [-2|\lambda|(2 N+n) t] \frac{(N+n-1) !}{N !}\right) \mathrm{d} \lambda \\
& \leqslant C t^{-n-2 k-1}\left(\Sigma(2 N+n)^{-2}\right) \leqslant C t^{-n-2 k-1} .
\end{aligned}
$$

This proves the estimate for the integral $I$.

Next consider $J$. Let us write $w=(z, s)$. We observe that

$$
\begin{aligned}
J & \leqslant C t^{-2 k} \iint\left(s^{2}+|z|^{4}\right)^{k}\left|F_{t}(z, s)\right|^{2} \mathrm{~d} z \mathrm{~d} s \\
& =C t^{-2 k} \iint\left|\left(i s-|z|^{2}\right)^{k} F_{t}(z, s)\right|^{2} \mathrm{~d} z \mathrm{~d} s .
\end{aligned}
$$

If we can show that the integral in (30) is bounded by $t^{-n-1}$ then we are done. If we write the Fourier transform of $G=\left(i s-r^{2}\right)^{k} F_{t}(z, s)$ in the form

$$
\hat{G}(\lambda)=\sum_{N=0}^{\infty} R_{N}\left(\lambda,\left(i s-|z|^{2}\right)^{k} F_{z}\right) P_{N}(\lambda)
$$

then we need to show that

$$
\int_{N=0}^{\infty}\left|R_{N}\left(\lambda,\left(i s-r^{2}\right)^{k} F_{t}\right)\right|^{2} \frac{(N+n-1) !}{N ! \cdot}|\lambda|^{n} \mathrm{~d} \lambda \leqslant C t^{-n-1}
$$

where we have set $|z|^{2}=r^{2}$.

Let us write

$$
\psi(N, \lambda)=(-1)^{k}(2 N+n)^{k}|\hat{\lambda}|^{k} \exp [-(2 N+n)|\lambda| t] \varphi((2 N+n)|\lambda|)
$$

so that $R_{N}\left(\lambda, F_{t}\right)=\psi(N, \lambda)$. We define $\psi_{k}(N, \lambda)$ to be $R_{N}\left(\lambda,\left(i s-r^{2}\right)^{k} F_{t}\right)$. Then the following estimate is valid.

Lemma 5.1. Under the hypothesis of the theorem there is an $\varepsilon>0$ such that

$$
\left|\psi_{k}(N, \lambda)\right| \leqslant C \exp [-\varepsilon(2 N+n)|\lambda| t .
$$

If we use (32) in (29) then the estimate $J \leqslant t^{-n-2 k-1}$ is immediate. So we proceed to prove Lemma 5.1 .

Recall the definition of $R_{N}(\lambda, f)$ for a zonal function $f$.

$$
R_{N}(\lambda, f)=C_{n} \frac{N !}{(N+n-1) !} \int_{0}^{\infty} f(r, \lambda) L_{N}^{n-1}\left(2|\lambda| r^{2}\right) \exp \left(-|\lambda| r^{2}\right) r^{2 n-1} \mathrm{~d} r
$$

where $\tilde{f}(r, \lambda)$ is the Euclidean Fourier transform of $f$ in the $s$ variable. We will prove 
(32) when $\lambda>0$. The case $\lambda<0$ is completely similar.

Since $($ isf $) \tilde{f}(r, \lambda)=(\mathrm{d} / \mathrm{d} \lambda) \tilde{f}(r, \lambda)$ we obtain

$$
\begin{aligned}
R_{N}(\lambda, i s f)= & \frac{\mathrm{d}}{\mathrm{d} \lambda} R_{N}(\lambda, f)-C_{n} \frac{N !}{(N+n-1) !} \int_{0}^{\infty} f(r, \lambda) \\
& \times \frac{\mathrm{d}}{\mathrm{d} \lambda}\left\{L_{N}^{n-1}\left(2 \lambda r^{2}\right) \exp \left(-\lambda r^{2}\right)\right\} r^{2 n-1} \mathrm{~d} r .
\end{aligned}
$$

Now

$$
\begin{aligned}
& \frac{\mathrm{d}}{\mathrm{d} \lambda}\left(L_{N}^{n-1}\left(2 \lambda r^{2}\right) \exp \left(-\lambda r^{2}\right)\right. \\
& \quad=2 r^{2} \frac{\mathrm{d}}{\mathrm{d} r} L_{N}^{n-1}\left(2 \lambda r^{2}\right) \exp \left(-\lambda r^{2}\right)-r^{2} L_{N}^{n-1}\left(2 \lambda r^{2}\right) \exp \left(-\lambda r^{2}\right)
\end{aligned}
$$

Using the recursion formula (see [8])

$$
r \frac{\mathrm{d}}{\mathrm{d} r} L_{N}^{n-1}(r)=N L_{N}^{n-1}(r)-(N+n-1) L_{N-1}^{n-1}(r)
$$

a simple calculation shows that

$$
R_{N}(\lambda, i s f)=\frac{\mathrm{d}}{\mathrm{d} \lambda} R_{N}(\lambda, f)-\frac{N}{\lambda}\left(R_{N}(\lambda, f)-R_{N-1}(\lambda, f)\right)+R_{N}\left(\lambda, r^{2} f\right) .
$$

Thus we have obtained the formula

$$
\psi_{1}(N, \lambda)=\frac{\partial \psi}{\partial \lambda}-\frac{N}{\lambda}(\psi(N, \lambda)-\psi(N-1, \lambda))
$$

Since $\psi(N, \lambda)=\psi((2 N+n) \lambda)$ we can write (35) in the form

$$
\psi_{1}(N, \lambda)=\frac{1}{2} \frac{n}{\lambda} \frac{\partial \psi}{\partial N}+\frac{N}{\lambda}\left(\frac{\partial \psi}{\partial N}-\Delta \psi\right),
$$

where $\Delta \psi(N, \lambda)=\psi(N, \lambda)-\psi(N-1, \lambda)$. Define the operators $S, D$ and $T$ by

$$
S \psi=\frac{\partial \psi}{\partial N}, \quad D \psi=\frac{\partial \psi}{\partial N}-\Delta \psi, \quad T \psi=N D \psi
$$

So, we have

$$
\psi_{1}(N, \lambda)=\lambda^{-1}\left(\frac{n}{2} S+T\right) \psi(N, \hat{\lambda})
$$

From this formula we can conclude that

$$
\psi_{k}(N, \lambda)=\lambda^{-k} \sum_{i+j+m=k} a_{i j m} S^{i} T^{j} S^{m} \psi(N, \lambda)
$$

Now we observe that $S^{m} \psi(N, \lambda)=\psi^{(m)}((2 N+n) \lambda)(2 \lambda)^{m}$ and by hypothesis of the thèrem $S^{m}$ in essence brings a factor $(2 N+n)^{-m}$. We will show that $T^{j}$ also does 
the same thing. Then each term in the sum (38) will behave like $\lambda^{-k}(2 N+n)^{-k} \psi(N, \lambda)$. Recalling the definition of $\psi(N, \lambda)$ we see that

$$
\left|\psi_{k}(N, \lambda)\right| \leqslant C \exp [-\varepsilon(2 N+n) \lambda t]
$$

as desired.

For the operators $T^{j}$ the following formula is valid.

\section{Lemma 5.2.}

$$
T^{j} \psi=\sum C_{p q m} N^{p} D^{q}\left(\Delta^{m} \psi\right)
$$

where the sum is extended over all $p, q, m$ satisfying the relation $j+p \leqslant 2 q+m \leqslant 2 j$.

Proof. We prove this lemma by induction. We first observe that from the definition of $T$, the lemma is trivially valid for $j=1$. Now assume the lemma true for some $j$ and consider $T^{j+1} \psi$

$$
T^{j+1} \psi=\sum C_{p q m} N D\left(N^{p} D^{q}\left(\Delta^{m} \psi\right)\right)
$$

where $j+p \leqslant 2 q+m \leqslant 2 j$. We need a formula for $D\left(N^{p} D \psi\right)$.

We claim that

$$
D\left(N^{p} D \psi\right)=N^{p} D^{2} \psi+\sum_{i=0}^{p-1} a_{i} N^{i} D(\Delta \psi)+\sum_{i=0}^{p-2} b_{i} N^{i} D \psi
$$

Assuming the claim for a moment we have

$$
\begin{aligned}
T^{j+1} \psi= & \sum_{p, q, m} C_{p q m} N^{p+1} D^{q+1}\left(\Delta^{m} \psi\right)+\sum_{p, q, m} C_{p q m} \sum_{i=0}^{p-1} a_{i} N^{i+1} D^{q}\left(\Delta^{m+1} \psi\right) \\
& +\sum_{p, q, m} C_{p q m} \sum_{i=0}^{p-2} b_{i} N^{i+1} D^{q}\left(\Delta^{m} \psi\right) .
\end{aligned}
$$

From this formula it is clear that $T^{j+1} \psi$ is of the desired form.

To prove the claim we first observe that

$$
\Delta(\varphi \psi)(N)=\Delta \varphi(N) \psi(N)+\varphi(N-1) \Delta \psi(N) .
$$

In view of this formula

$$
\Delta\left(N^{p} D \psi\right)=\Delta\left(N^{p}\right) D \psi+(N-1)^{p} D(\Delta \psi) .
$$

We also have

$$
\begin{aligned}
\Delta\left(N^{p}\right) & =N^{p}-(N-1)^{p}=p N^{p-1}-\sum_{i=0}^{p-2} b_{i} N^{i} \\
(N-1)^{p} D(\Delta \psi) & =N^{p} D(\Delta \psi)-\sum_{i=0}^{p-1} a_{i} N^{i} D(\Delta \psi) \\
\frac{\partial}{\partial N}\left(N^{p} D \psi\right) & =p N^{p-1} D \psi+N^{p} D\left(\frac{\partial \psi}{\partial N}\right) .
\end{aligned}
$$


From (42)-(45) it follows that

$$
D\left(N^{p} D \psi\right)=N^{p} D^{2} \psi+\sum_{i=0}^{p-1} a_{i} N^{i} D(\Delta \psi)+\sum_{i=0}^{p-2} b_{i} N^{i} D \psi
$$

This proves the claim.

Finally we will show that the action of $T^{j}$ has the desired properties. We have

$$
T^{j} \psi=\sum C_{p q m} N^{p} D^{q}\left(\Delta^{m} \psi\right)
$$

where $p+j \leqslant 2 q+m \leqslant 2 j$. Now using Taylor's formula with integral form of remainder we can write

$$
D \psi(N)=\int_{0}^{1} t \psi^{\prime \prime}(N-1+t, \lambda) \mathrm{d} t
$$

where the primes stand for the derivatives with respect to $N$. From (48) it is clear that the action of $D$ is to bring down the factor $N^{-2}$. An iteration will show that $D^{q}$ will bring down the factor of $N^{-2 q}$ when applied to $\psi$. Since $\Delta^{m} \psi$ brings down $N^{-m}$ the formula (47) shows that $T^{j}$ acting on $\psi$ brings down the factor

$$
\sum C_{p q m} N^{p} N^{-2 q-m} \text {. }
$$

Since $p+j \leqslant 2 q+m$, essentially $T^{j}$ brings down a factor of $N^{-j}$ as required.

\section{References}

[1] Coifman $\mathbf{R}$ and Weiss $\mathbf{G}$, Analyse harmonique non commutative sur certaines espaces homogenes, in Lecture notes in Math. (Berlin: Springer-Verlag) Vol. 242 (1971)

[2] Folland G, Subelliptic estimates and function spaces on nilpotent Lie groups, Ark. Math. 13 (1975) 161-207

[3] Geller D, Fourier analysis on the Heisenberg group, J. Funct. Anal. 36 (1980) 205-254

[4] Mauceri G, Zonal multipliers on the Heisenberg group, Pacific J. Math. 95 (1981) 143-159

[5] de Michele L and Mauceri G, $H^{p}$ muitipliers on stratified groups, Annali Mat. Pura. Appl. 148 (1987) 353-366

[6] Stein E M, Topics in harmonic analysis (Princeton: University Press) 63 Ann. Math. Stud. (1971)

[7] Strichartz R S, Multipliers for spherical harmonic expansions, Trans. A.M.S. 167 (1972) 115-124

[8] Szego G, Orthogonal polynomials, Am. Math. Soc. Colloq. Pub. Providence (1967) vol. 23

[9] Thangavelu S, Multipliers for Hermite expansions, Revista Math. Ibero. 3 (1987) 1-24

[10] Thangavelu S, Littlewood-Paley-Stein theory on $C^{n}$ and Weyl multipliers Revista Mat. Ibero. 6 (1990) 75-90 